hep-th/0004206

\title{
Self-tuning in an Outgoing Brane Wave Model
}

\author{
Gary T. Horowitz ${ }^{1}$ Ian $\mathrm{Low}^{2,3}$, and A. Zee ${ }^{1,2}$ \\ ${ }^{1}$ Department of Physics, \\ University of California, Santa Barbara, CA 93106 \\ ${ }^{2}$ Institute for Theoretical Physics, \\ University of California, Santa Barbara, CA 93106 \\ ${ }^{3}$ Department of Physics, \\ Carnegie Mellon University, Pittsburgh, PA 15213
}

\begin{abstract}
We introduce a new brane-world model in which the bulk solution consists of outgoing plane waves. This is an exact solution to string theory with no naked singularities. The recently discussed self-tuning mechanism to cancel the cosmological constant on a brane is naturally incorporated. We show that even if the vacuum energy on the brane changes, e.g. due to a phase transition, the brane geometry remains insensitive to the local vacuum energy. We also consider the static self-tuning branes introduced earlier, and find a solution in which the brane geometry starts to contract when the vacuum energy on the brane changes.
\end{abstract}




\section{INTRODUCTION}

The idea that our universe is embedded in a higher dimensional world has received a great deal of renewed attention over the last two years [1,2]. Of particular interest is the fact that this opens up new approaches to solving the cosmological constant problem (see,

e.g., [3 5]) though related ideas were pursued some time ago [6 8]. In particular, [4.5] show that in a five dimensional theory of gravity coupled to a scalar field, the brane geometry can remain flat, independent of the value of the cosmological constant on the brane. This is known as "self-tuning". There has been extensive discussion of this idea for static branes [9]. However, a natural question to ask is whether the same self-tuning feature will remain in a dynamical context where the vacuum energy on the brane changes, e.g., due to a phase transition.

Dynamical discussions of our universe as a brane suffer from the awkward feature that evolution requires not just initial data on the brane, but also initial data for the bulk fields as well. Thus, there are a large number of degrees of freedom that we do not have direct access to. The simplest, and most natural assumption is that the these bulk degrees of freedom simply respond to motion on the brane. In other words, there are no incoming bulk waves, but only outgoing waves. We construct and study this "outgoing brane wave model". It has a number of desirable features: (1) The metric in the bulk is an exact plane wave. Since the curvature is null, all higher order corrections to Einstein's equation constructed from higher powers of the Riemann tensor automatically vanish. Hence, these metrics are exact solutions to string theory to all order in $\alpha^{\prime}[10,11]$. (2) The geometry on the brane is not flat, but is typically given by an expanding Robertson-Walker cosmology円. However, the rate of expansion is independent of the value of the cosmological constant, so the self-tuning feature is incorporated. This continues to hold even when the vacuum energy on the brane changes due to a phase transition. (3) There are no naked singularities. Instead, there are

\footnotetext{
${ }^{1}$ For other discussions of brane-world cosmology see [12].
} 
null singularities in the past which extend from the big bang on the brane. (4) As mentioned above, this is a deterministic model in which the bulk geometry is determined solely by the brane.

Even though one does not need to specify boundary conditions at the null singularity, it still cuts off space in the fifth dimension. So the proper distance to the singularity on appropriate spacelike surfaces is finite, and the local four dimensional Newton's constant will be nonzero. However, it is also time dependent. This is a problem with this model which is currently under investigation. Perhaps by adding a bulk cosmological constant one could localize gravity near the brane [2] and stabilize the effective Newton's constant.

We also find exact, time dependent solutions to the bulk equations which are more general than plane waves. These solutions can be used to study a phase transition in which the vacuum energy changes on an initially static, Poincare invariant brane. We find a solution in which the brane becomes time dependent after the transition. Nevertheless, it remains an open question whether or not the brane becomes time dependent in all solutions with an initially static brane.

After discussing the basic equations of motion in the next section, we introduce our outgoing brane wave model in section III and study its properties. We then consider more general time dependent solutions in section IV and use them to study phase transitions on initially static branes in section $\mathrm{V}$.

\section{EQUATIONS OF MOTION}

Following [4.5], we take the low energy effective action to be

$$
S=\int d^{5} x \sqrt{-G}\left[R-\frac{4}{3}(\nabla \varphi)^{2}\right]+\int d^{4} x \sqrt{-g} e^{b \varphi} \mathcal{L}_{4 D}
$$

Gravity and the dilaton field $\varphi$ live in a 5-dimensional world (with coordinates $t, x^{i}$, and $y$ ) and are coupled to a thin 4-dimensional brane whose position is taken to be at $y=0$. The metric $g_{\mu \nu}=\delta_{\mu}^{M} \delta_{\nu}^{N} G_{M N}$ is the 4-dimensional metric induced on the brane, and $\mathcal{L}_{4 D}$ denotes 
the Lagrangian of the 4-dimensional world. (Here $M, N=0,1,2,3,5 ; \mu, \nu=0,1,2,3$; and we have set $16 \pi G_{5}=1$.) We take $b$ as a free phenomenological parameter which ultimately may be determined by string theory. We will assume the geometry on the brane is homogeneous and isotropic, so the stress energy tensor must take the form of a perfect fluid:

$$
\begin{aligned}
T_{\nu}^{\mu} & =e^{b \varphi} \operatorname{diag}\left(-\rho_{T}, P_{T}, P_{T}, P_{T}\right) \\
& =-e^{b \varphi} V \delta^{\mu}{ }_{\nu}+e^{b \varphi} \operatorname{diag}(-\rho, P, P, P)
\end{aligned}
$$

where we have set $\rho_{T}=\rho+V$ and $P_{T}=P-V$. With this form we describe a 4-dimensional world containing a vacuum energy $V$ and a perfect fluid with energy density $\rho$ and pressure $P$. We will treat $V, \rho$, and $P$ as phenomenological functions of $t$.

Einstein's equations read

$$
R_{M N}-\frac{1}{2} G_{M N} R=\frac{4}{3}\left[\nabla_{M} \varphi \nabla_{N} \varphi-\frac{1}{2} G_{M N}(\nabla \varphi)^{2}\right]+\frac{1}{2} \sqrt{\frac{g}{G}} T_{\mu \nu} \delta_{M}^{\mu} \delta_{N}^{\nu} \delta(y)
$$

with $g$ and $G$ the determinant of $g_{\mu \nu}$ and $G_{M N}$ respectively. The equation of motion for the scalar field has a source involving the the four dimensional Lagrangian $\mathcal{L}_{4 D}$. The Lagrangian for a perfect fluid (with pressure determined in terms of the energy density) is simply $\mathcal{L}=-\rho$ [13. So if we model the matter on the brane by a vacuum energy and perfect fluid, we have $\mathcal{L}_{4 D}=-(V+\rho)$. The dilaton equation is thus

$$
\frac{8}{3} \nabla^{2} \varphi=\sqrt{\frac{g}{G}} b e^{b \varphi}(V+\rho) \delta(y)
$$

We wish to consider solutions depending on both $t$ and $y$, which are homogeneous and isotropic in the three transverse directions. It will be convenient to adopt "conformal gauge" in the $t, y$ directions, and write the metric in the form

$$
\begin{aligned}
d s^{2} & =e^{2 A(t, y)}\left(-d t^{2}+d y^{2}\right)+e^{2 B(t, y)}\left(d x_{1}^{2}+d x_{2}^{2}+d x_{3}^{2}\right) \\
& =-e^{2 A(u, v)} d u d v+e^{2 B(u, v)}\left(d x_{1}^{2}+d x_{2}^{2}+d x_{3}^{2}\right)
\end{aligned}
$$

where as usual $u \equiv t-y$ and $v \equiv t+y$.

The easiest way to solve Einstein's equation ([1.3) and the dilaton equation (II.4) is to first solve them in the bulk, that is, away from the brane at $y=0$. We will solve these 
equations for $A(t, y), B(t, y)$, and $\varphi(t, y)$ for $y>0$ and $y<0$ separately and then match the solutions across the brane. We find it convenient to use the $(u, v)$ light cone coordinates to solve the equations in the bulk, and the $(t, y)$ coordinates to do the matching across the brane.

Using the metric ([1.5) we obtain the following bulk equations of motion

$$
\begin{aligned}
B_{, u v}+3 B_{, u} B_{, v} & =0 \\
2 \varphi_{, u v}+3\left(B_{, u} \varphi_{, v}+B_{, v} \varphi_{, u}\right) & =0 \\
A_{, u} B_{, u}-\frac{1}{2}\left(B_{, u}^{2}+B_{, u u}\right)-\frac{2}{9} \varphi_{, u}^{2} & =0 \\
A_{, v} B_{, v}-\frac{1}{2}\left(B_{, v}{ }^{2}+B_{, v v}\right)-\frac{2}{9} \varphi_{, v}{ }^{2} & =0 \\
2 \varphi_{, u} \varphi_{, v}+3\left(A_{, u v}+2 B_{, u v}+3 B_{, u} B_{, v}\right) & =0
\end{aligned}
$$

Here (II.6) is the $u v$ component of Einstein's equation. It is independent of $\varphi$ since the dilaton is essentially a two dimensional scalar field and the $u v$ component of the stress energy tensor is like the trace of the two dimensional stress tensor which vanishes. ([II.7) is the dilaton equation of motion. Both of these first two equations are independent of $A$ essentially because the two dimensional wave equation is conformally invariant. ([1.8) is the $u u$ component of the Einstein equation, (II.9) is the $v v$ component, and (II.10) the $i i$ component. This last equation is a consequence of the first four by virtue of the Bianchi identity.

The jump conditions for the metric at the brane can be obtained from the usual thin shell conditions of general relativity [14]. The unit outward normal to the surface $y=0$ is $n=e^{-A} \partial / \partial y$. The extrinsic curvature $K_{\mu \nu}=\nabla_{\mu} n_{\nu}$ is

$$
K_{t t}=-e^{A} A_{, y} \quad K_{i j}=e^{2 B-A} B_{, y} \delta_{i j}
$$

and its trace is $K=K_{\mu}{ }^{\mu}=e^{-A}\left(A_{, y}+3 B_{, y}\right)$. In general, if $\left.K_{\mu \nu}\right]$ is the jump in the extrinsic curvature across the surface $y=0$, the stress tensor on the brane is given by

\footnotetext{
${ }^{2}$ They can also be obtained by writing out (II.3) and integrating across $y=0$ at fixed $t$.
} 


$$
\left.\left.T_{\mu \nu}=-\frac{1}{8 \pi G_{5}}\left(K_{\mu \nu}\right]-g_{\mu \nu} K\right]\right)
$$

(For any function $F(t, y)$, we use the notation $F]=F\left(t, 0^{+}\right)-F\left(t, 0^{-}\right)$and $F \mid=F(t, 0)$.) Using the fact that we have set $16 \pi G_{5}=1$, and the form of the stress tensor ([1.2), we obtain the matching conditions

$$
\begin{aligned}
\left.-6 \frac{\partial B}{\partial y}\right] & =e^{A+b \varphi}\left|\rho_{T}=e^{A+b \varphi}\right|(V+\rho), \\
\left.6 \frac{\partial A}{\partial y}\right] & =e^{A+b \varphi} \mid\left(2 \rho_{T}+3 P_{T}\right) \\
& =e^{A+b \varphi} \mid(2 \rho+3 P-V) \\
\left.\frac{8}{3} \frac{\partial \varphi}{\partial y}\right] & =b e^{A+b \varphi} \mid(V+\rho) .
\end{aligned}
$$

where the matching condition for the dilaton is obtained by integrating (III.4) at fixed $t$ across $y=0$.

It is worth emphasizing that the matching conditions have to be satisfied at any instant in $t$. In other words, both sides in the matching conditions ([1.13), (III.14) and (ㅍ.15) are functions of $t$, as opposed to be just constants in the static case. Comparing (III.15) and (11.13) we find

$$
\left.\left.\varphi_{, y}\right]=-\frac{9}{4} b \quad B_{, y}\right]
$$

which is a rather strong constraint since $b$ is a constant. In many of our solutions this condition forces $\varphi$ to be proportional to $B$.

Note that in the 4-dimensional universe on the brane, the metric is given by

$$
d s^{2}=-e^{2 A(t, 0)} d t^{2}+e^{2 B(t, 0)}\left(d x_{1}^{2}+d x_{2}^{2}+d x_{3}^{2}\right)
$$

which can be written in the standard form of a Robertson-Walker universe

$$
d s^{2}=-d \tau^{2}+a(\tau)^{2}\left(d x_{1}^{2}+d x_{2}^{2}+d x_{3}^{2}\right)
$$

with

$$
\begin{aligned}
\tau & =\int d t e^{A(t, 0)}, \\
a(\tau) & =e^{B(t(\tau), 0)} .
\end{aligned}
$$




\section{OUTGOING BRANE WAVE MODEL}

There is a simple class of solutions to the bulk equations ([I.6) - ([I.10) which consists of taking $A, B$, and $\varphi$ to depend only on $u$. The five field equations then collapse to just one

$$
A_{, u} B_{, u}-\frac{1}{2}\left(B_{, u}^{2}+B_{, u u}\right)-\frac{2}{9} \varphi_{, u}^{2}=0
$$

This corresponds to a plane wave propagating to the right. It may appear that this class of solutions has two arbitrary functions of $u$, but one of them can be absorbed by reparametrizing $u$. The remaining free function can be taken to be the amplitude of the dilaton field $\varphi$. There are no independent gravitational degrees of freedom since we have assumed isotropy in the transverse $x^{i}$ space. (Gravitational plane waves would expand some directions while contracting others.) There are clearly analogous solutions depending only on $v$ representing plane waves moving to the left.

We now construct a solution of the full equations (II.3) and (II.4) by matching a solution depending only on $u$ (for $y>0$ ) to a solution depending only on $v$ (for $y<0$ ). The result is a solution in which the bulk spacetime consists of plane waves moving away from the brane on both sides. We will call this the outgoing brane wave model.

The matching conditions at $y=0$ can be solved as follows. It will be convenient to write

$$
B(u, v)=\log h(u)
$$

on the $y>0$ side. The continuity of $B$ implies that $B(u, v)=\log h(v)$ on the $y<0$ side.

Since, according to ( $(\mathbb{1 . 1 6})$, the jump in $\varphi_{, y}$ must be proportional to the jump in $B_{, y}$ for all $t, \varphi$ itself must be proportional to $B$.

$$
\varphi(u, v)=-\frac{9}{4} b \log h(u)
$$

for $y>0$ and $\varphi(u, v)=-\frac{9}{4} b \log h(v)$ for $y<0$. Note that an additive constant in $\varphi(u, v)$ can be absorbed by scaling $h$ and the consequent additive constant in $B$ can be absorbed 
by scaling $x^{i}$. We can now immediately integrate ([II.1) to obtain?

$$
\begin{aligned}
A(u, v) & =\frac{1}{2}\left(1+\frac{9}{4} b^{2}\right) B(u, v)+\frac{1}{2} \log B_{, u}(u, v) \\
& =\frac{9}{8} b^{2} \log h(u)+\frac{1}{2} \log h^{\prime}(u)
\end{aligned}
$$

for $y>0$ and $A(u, v)=\frac{9}{8} b^{2} \log h(v)+\frac{1}{2} \log h^{\prime}(v)$ for $y<0$. An additive constant can be absorbed by scaling $u$ and $v$.

The function $h$ is determined once one picks an equation of state for the matter on the brane. In other words, the equation of states fixes both the amplitude of the bulk waves, and the dynamics of the brane geometry. Let

$$
P_{T}=\gamma \rho_{T}
$$

where $\gamma$ is a constant, and $P_{T}$ and $\rho_{T}$ are the total pressure and energy density. (A cosmological constant corresponds to $\gamma=-1$.) Then the matching conditions ([I.13) and (II.14) imply that the jump in $A_{, y}$ is proportional to the jump in $B_{, y}$. Since this must hold for all time, this implies

$$
A=-(2+3 \gamma) B+k
$$

for some constant $k$. This yields a first order equation for $h$ which can be integrated explicitly.

To illustrate some of the features of this model we start with a particularly simple special case. Inspection of (III.1) shows that we can choose $\varphi, A$, and $B$ to be linear functions of $u$ on the $y>0$ side and linear functions of $v$ on the $y<0$ side respectively. This corresponds to setting

$$
h(t)=e^{\lambda t}
$$

where we will assume the constant $\lambda$ is positive. Thus, on the $y>0$ side, $B=\lambda u$, $\varphi=-\frac{9}{4} b \lambda u$, and

\footnotetext{
${ }^{3}$ Throughout this paper it is understood that all functions inside the logarithm have absolute value signs.
} 


$$
A=\frac{1}{2}\left(1+\frac{9}{4} b^{2}\right) \lambda u+\frac{1}{2} \log \lambda
$$

and similarly on the $y<0$ side. We now set $b= \pm \frac{2}{3}$. (The case of general $b$ will be considered below.) Then $A=B+$ const, and it follows from ([II.6) that $\gamma=-1$ so the stress energy on the brane is a pure cosmological constant. From (【I.13), the vacuum energy is

$$
V=12 \lambda^{\frac{1}{2}}
$$

The bulk metric is

$$
d s^{2}=e^{2 \lambda(t-y)}\left[-\lambda d t^{2}+\lambda d y^{2}+d x_{i} d x^{i}\right]
$$

for $y>0$ and

$$
d s^{2}=e^{2 \lambda(t+y)}\left[-\lambda d t^{2}+\lambda d y^{2}+d x_{i} d x^{i}\right]
$$

for $y<0$. Changing to cosmological time $\tau=e^{\lambda t} / \sqrt{\lambda}$, we see that the metric on the brane at $y=0$ has the Robertson-Walker form $d s^{2}=-d \tau^{2}+\lambda \tau^{2} d x_{i} d x^{i}$ which after scaling $x^{i}$ gives

$$
d s^{2}=-d \tau^{2}+\tau^{2} d x_{i} d x^{i}
$$

Thus, with a constant vacuum energy $V$, we have a universe with the scale factor growing linearly $a(\tau)=\tau$ rather the usual exponential growth. It is important to note that the expansion rate is independent of the value of the vacuum energy (as long as it is nonzero). Thus this solution has the self-tuning feature described in [4, 可].

Unlike the static solutions discussed in [4,5], this solution has no naked timelike singularity. However there is a null singularity at $u=-\infty$ on the right of the brane and at $v=-\infty$ on the left. Null geodesics from these singularities reach the brane in finite affine parameter so they are not really at infinity. This is most easily seen by introducing a new coordinate $U=e^{2 \lambda u} / 2$ so the metric for $y>0$ becomes

$$
d s^{2}=-d U d v+2 U d x_{i} d x^{i}
$$




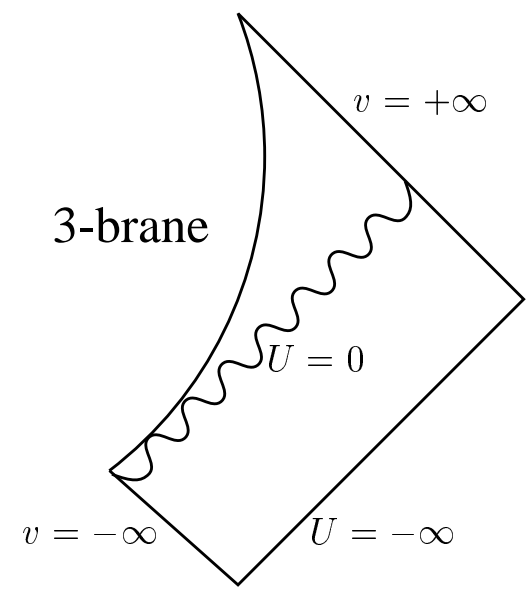

FIG. 1. The causal structure on the right of the brane for the case $b= \pm \frac{2}{3}$. The brane never actually hits the singularity in the bulk.

The singularity is now at $U=0$. The brane is located at $u=v$ or $U=e^{2 \lambda v} / 2$, so the brane never actually hits the singularity, but instead becomes asymptotically null as $v \rightarrow-\infty$ (see Fig. 1). The geometry on the left of the brane is similar with the roles of $u$ and $v$ interchanged.

Since the null singularity cuts off space in the fifth direction, one has a nonzero effective four dimensional Newton's constant $G_{4}$. But since the distance of the singularity to the brane changes with time, $G_{4}$ will be time dependent. This is the main drawback of the outgoing brane wave model. It can perhaps be cured by modifying the bulk Lagrangian to try to localize gravity as in the Randall-Sundrum scenario.

As discussed in [4], the value of $b$ that we have chosen, $b=\frac{2}{3}$, arises naturally in string theory since then ([I.1) is just the Einstein frame action arising from a string frame source proportional to $e^{-2 \varphi}$. For our solution, the corresponding string metric $\tilde{G}_{M N}=e^{4 \varphi / 3} G_{M N}$ is flat! The bulk solution is simply a linear dilaton vacuum where the dilaton is proportional to a null coordinate. This is well known to be an exact solution to string theory [15]. The brane geometry is also flat in the string frame.

The general solution for $h$ given an arbitrary dilaton coupling to the brane $b$ and arbitrary equation of state $P_{T}=\gamma \rho_{T}$ is easy to construct. From ([II.4) and (III.6) we get a first order 
equation for $h$ with solution

$$
h(u)=(\lambda u)^{4 /\left(20+24 \gamma+9 b^{2}\right)}
$$

for $y>0$ where $\lambda$ is a positive constant related to $k$ in (III.6). The solution for $y<0$ is identical with $u$ replaced by $v$. This is valid whenever the exponent is finite. For the special case considered above $(\gamma=-1$ and $b= \pm 2 / 3)$ the exponent diverges and the solution for $h$ is not a power law, but rather an exponential as we saw. The metric on the brane turns out to be

$$
d s^{2}=-d \tau^{2}+\tau^{8 /\left[12(1+\gamma)+9 b^{2}\right]} d x_{i} d x^{i}
$$

after rescaling the $x_{i}$. In particular, for a pure cosmological constant $(\gamma=-1)$ the brane geometry is

$$
d s^{2}=-d \tau^{2}+\tau^{8 /\left(9 b^{2}\right)} d x_{i} d x^{i}
$$

Thus, for any nonzero coupling $b$, the expansion is a power law which is independent of the value of the vacuum energy. The value of this vacuum energy can be computed from (II.13). When there is no other matter on the brane $(\gamma=-1)$ one finds that the vacuum energy is indeed constant on the brane and given by

$$
V= \pm \frac{24}{\left|9 b^{2}-4\right|^{\frac{1}{2}}} \lambda^{\frac{1}{2}} .
$$

where the sign is the same as the sign of $9 b^{2}-4$. The causal structure of the resulting spacetime is shown in Fig. 2. In this case, the singularity is at $u=0$ and the brane hits the singularity at the time of the big bang.

The properties of this solution are similar to the case $b=\frac{2}{3}$ discussed above. (In fact, Fig. 2 accurately represents the causal structure of the complete spacetime in this case also.) It is even true that the bulk spacetime is still an exact solution to string theory. In terms of a new null coordinate $U$, the bulk metric becomes

$$
d s^{2}=-d U d v+U^{8 /\left(4+9 b^{2}\right)} d x_{i} d x^{i}
$$




\section{3-brane}

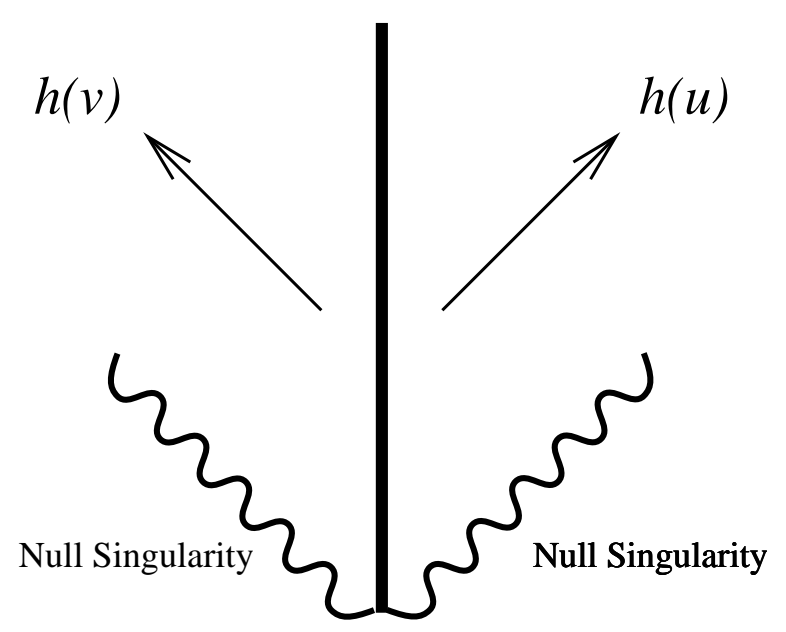

FIG. 2. The causal structure of the outgoing brane wave model

Surprisingly, this metric is independent of the equation of state parameter $\gamma$. However, the position of the brane in $U, v$ coordinates now depends on $\gamma$. The metric (III.18) has a covariantly constant null Killing vector $\ell=\partial / \partial v$ in addition to the translational symmetries in $x^{i}$. So it belongs to a general class of metrics known as exact plane waves. The Riemann tensor involves two powers of $\ell$ so all scalar curvature invariants vanish. Nevertheless, the singularity is real and results in infinite tidal forces. From the standpoint of string theory, since the curvature is null, all $\alpha^{\prime}$ corrections automatically vanish [11]. Moreover, one can show that the exact two dimensional beta functions of a sigma-model with this target space vanish, so this is an exact solution to string theory even nonperturbatively [10].

When $b=0$ (and $\gamma=-1$ ) (III.14) shows that $h=(\lambda u)^{-1}$. The bulk metric is then $d s^{2}=(\lambda u)^{-2}\left(-\lambda d u d v+d x_{i} d x^{i}\right)$ which is actually flat space in disguise. The metric on the brane is $-d \tau^{2}+e^{-2 \tau \sqrt{\lambda}} d x_{i} d x^{i}$ and we recover the usual de Sitter metric.

We now ask what happens if there is a transition from one value of the vacuum energy $V_{1}$ to another value $V_{2}$. Since a pure vacuum energy is always constant, to achieve this we must assume that the matter on the brane consists of both a vacuum energy and another component which we take to be radiation. Physically, some of the initial vacuum energy is 
converted to radiation which is then redshifted away leaving a smaller vacuum energy in the future. Since the two independent components of the stress energy tensor ([1.13) and (II.14) are determined in terms of $A, B$ and $\varphi$ and these three functions are determined in terms of $h$ via ([11.2), ([III.3), and ([11.4), we can compute $V(t)$ and $\rho(t)$ given any $h(t)$. Setting $P=\rho / 3$ we find

$$
V=\frac{3 h^{\frac{9}{8}} b^{2}}{\left|h^{\prime}\right|^{\frac{1}{2}}}\left[\left(3+\frac{9}{8} b^{2}\right) \frac{h^{\prime}}{h}+\frac{h^{\prime \prime}}{2 h^{\prime}}\right]
$$

and

$$
\rho=\frac{3 h^{\frac{9}{8}} b^{2}}{\left|h^{\prime}\right|^{\frac{1}{2}}}\left[\left(1-\frac{9}{8} b^{2}\right) \frac{h^{\prime}}{h}-\frac{h^{\prime \prime}}{2 h^{\prime}}\right]
$$

Here everything is evaluated on the brane so that $h$ stands for $h(t), h^{\prime}$ for $\frac{d h(t)}{d t}$, and so forth.

To show that the brane geometry remains independent of the value of the vacuum energy even after a phase transition, one must find a function $h(t)$ for which $\rho \geq 0$ everywhere, $\rho \rightarrow 0$ in the past and future, and $V$ approaches different constants in the past and future. Since ([II.16) is the only solution for pure vacuum energy, the geometry will expand at this rate both in the past and future. The only difference will be a constant rescaling of the $x^{i}$ coordinates. It is not hard to find a suitable function $h(t)$. For example, in the case $b= \pm \frac{2}{3}$, we can choose

$$
h(t)=\frac{e^{\lambda_{1} t}}{1+e^{\left(\lambda_{1}-\lambda_{2}\right) t}}
$$

with $\lambda_{1}>\lambda_{2}$. This describes a universe with pure vacuum energy $V_{1}=12 \lambda_{1}^{1 / 2}$ in the past and $V_{2}=12 \lambda_{2}^{1 / 2}$ in the future. During intermediate times there is radiation with energy density given by substituting ([11.21) into ([11.20). In Fig. 3 we show the time dependence of $V(t)$ and $\rho(t)$, assuming radiation only, for the case $\lambda_{1}=2.5, \lambda_{2}=1$ and $b=2 / 3$.

\section{GENERAL TIME DEPENDENT SOLUTIONS}

We now turn to more general time dependent solutions of the bulk equations of motion (II.6) -(【I.10). Remarkably, one can find explicit solutions which are much more general 

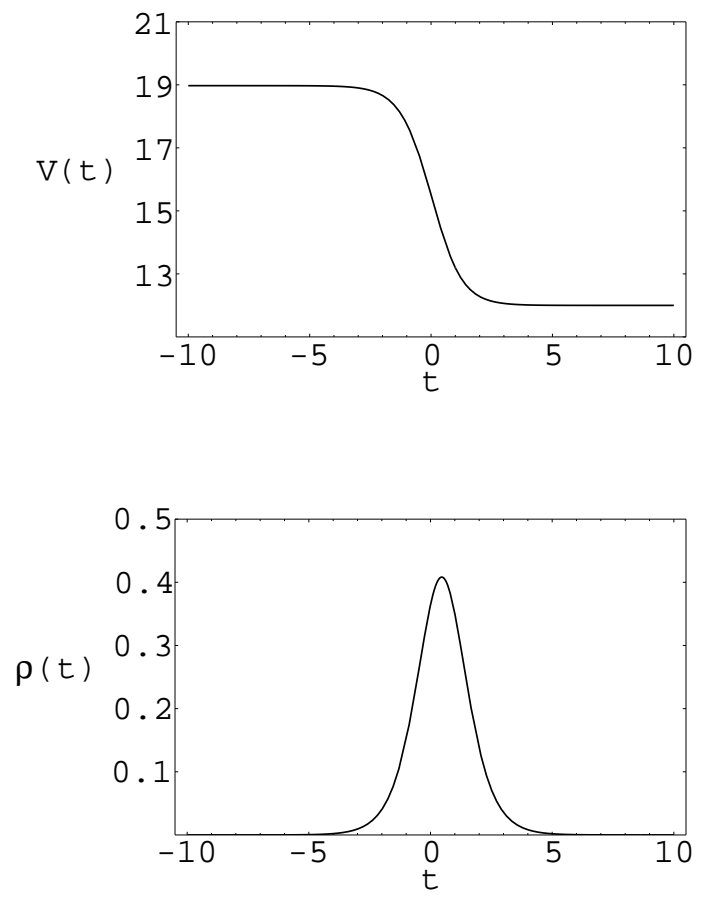

FIG. 3. Time dependence of $V(t)$ and $\rho(t)$ using $\lambda_{1}=2.5$ and $\lambda_{2}=1$ in (III.21) with $b=2 / 3$. We assume radiation only and thus $P=\rho / 3$.

than the plane waves discussed so far. One can then match these solutions on the brane to obtain solutions to the full field equations.

The key is to observe that (II.6) involves only $B$ and can be solved to give

$$
B(u, v)=\frac{1}{3} \log (f(u)+g(v))
$$

where $f$ and $g$ are two arbitrary functions. (A possible additive integration constant can always be absorbed by an overall scaling of $f$ and $g$.)

Given $B(u, v)$, we can solve the dilaton equation of motion (II.7). Using separation of variables, the general solution can be written

$$
\varphi(u, v)=\int d k \frac{c(k)}{\sqrt{(f(u)-k)(g(v)+k)}}
$$

A special case is obviously

$$
\varphi(u, v)=\frac{c}{\sqrt{(f(u)-k)(g(v)+k)}}
$$


For this case, we can solve ([I.8) and ([I.9) explicitly to find $A(u, v)$ with the result

$$
A(u, v)=-\frac{1}{3} \log (f(u)+g(v))+\frac{1}{2} \log \left(f^{\prime}(u) g^{\prime}(v)\right)-\frac{c^{2}}{12} \frac{(f(u)+g(v))^{2}}{((f(u)-k)(g(v)+k))^{2}}+a
$$

Another special solution for $\varphi$ which will be more useful in the following is

$$
\varphi(u, v)=\frac{\alpha}{3} \log (f(u)+g(v))
$$

and the associated solution for $A$ is

$$
A(u, v)=\left(\frac{2 \alpha^{2}}{9}-1\right) \frac{1}{3} \log (f(u)+g(v))+\frac{1}{2} \log \left(f^{\prime}(u) g^{\prime}(v)\right)+a
$$

with $\alpha$ and $a$ integration constants.

As an aside we mention one other special solution

$$
\varphi(u, v)=f(u)-g(v)
$$

and

$$
A(u, v)=-\frac{1}{3} \log (f(u)+g(v))+\frac{1}{2} \log \left(f^{\prime}(u) g^{\prime}(v)\right)+\frac{1}{3}(f(u)+g(v))^{2}+a
$$

This solution is not interesting for our purposes since the matching conditions ([1.13), ([1.14), and (II.15) can only be satisfied if the stress-energy tensor on the brane vanishes 1 .

\section{MATCHING TO AN INITIALLY STATIC SOLUTION}

We can now address the question of what happens if one starts with the static solutions discussed in [4,5] and then changes the vacuum energy. We begin by writing down the static solution with our form of metric. For our solutions to be independent of $t$, the functions $A$,

\footnotetext{
${ }^{4}$ Continuity of $\varphi$ and $B$ requires $f_{+}=f_{-}$and $g_{+}=g_{-}$(where the subscripts denote the solution for $y>0$ or $y<0$ ). From (II.13) - (II.15) we see that this leads to the conclusion that on the brane $\rho_{T}=P_{T}=0$.
} 
$B$, and $\varphi$ can depend on $(v-u)$ only. Since we want the metric on the brane to be Poincare invariant, we set $A=B$. With these restrictions, the bulk equations (II.6) -(ㅍ.10) reduce to two equations:

$$
\begin{gathered}
A^{\prime \prime}(y)+3 A^{\prime}(y)^{2}=0, \\
9 A^{\prime}(y)^{2}-\varphi^{\prime}(y)^{2}=0,
\end{gathered}
$$

We wish to solve these equations for $y>0$ and $y<0$ separately and then match at $y=0$. Denoting the solutions in the two regions by subscripts + or - , we have for $y>0$

$$
A(y)=\frac{1}{3} \log \left(y_{+}-y\right)+a_{+},
$$

and for $y<0$

$$
A(y)=\frac{1}{3} \log \left(y+y_{-}\right)+a_{-}
$$

where $y_{ \pm}$and $a_{ \pm}$are constants of integration. In order to have a finite 4D Planck mass [4.5] are forced to have naked singularities at $y=y_{+}$and $y=-y_{-}$to cut off spacetime. So the relevant region of spacetime is $-y_{-}<y<y_{+}$. Making the appropriate coordinate transformation we see that these agree with the solutions given in [1] which we will refer to as KSS. From the dilaton equation (V.2), we have the freedom to choose on either side of the brane $\varphi=+3 A$ or $\varphi=-3 A$ up to a constant. Let us fix

$$
\varphi(y)=\log \left(y_{+}-y\right)+d_{+}
$$

for $y_{+}>y>0$. In the nomenclature of KSS, for $0>y>-y_{-}$the choice

$$
\varphi(y)=-\log \left(y+y_{-}\right)+d_{-}
$$

is known as a type I solution, while the choice

$$
\varphi(y)=\log \left(y+y_{-}\right)+d_{-}
$$

is known as type II. The continuity of $\varphi$ and $A$ at $y=0$ determines $d_{+}$and $a_{+}$in terms of the other constants. 
Since we have set $A=B$, the matching conditions ([I.13) and ([I.14) imply that the stress-energy tensor on the brane takes the form of a pure vacuum energy density, with

$$
\left.-6 A_{, y}\right]=2\left(\frac{1}{y_{+}}+\frac{1}{y_{-}}\right)=e^{A+b \varphi} \mid V
$$

The matching condition for $\varphi(\underline{11.15)}$ implies

$$
\left.\frac{8}{3} \varphi_{, y}\right]=-\frac{8}{3}\left(\frac{1}{y_{+}}-\frac{1}{y_{-}}\right)=b e^{A+b \varphi} \mid V
$$

for type I and

$$
\left.\frac{8}{3} \varphi_{, y}\right]=-\frac{8}{3}\left(\frac{1}{y_{+}}+\frac{1}{y_{-}}\right)=b e^{A+b \varphi} \mid V
$$

for type II. Taking the ratio of $\left.\varphi^{\prime}\right]$ and $\left.A^{\prime}\right]$ we see that type $\mathrm{I}$ is allowed only if $b \neq \pm \frac{4}{3}$ (assuming that both $y_{+}$and $y_{-}$are finite) while type II is allowed only if $b=-\frac{4}{3}$. The solution for $b=+\frac{4}{3}$ is obtained from the type II solution by simply changing the sign of $\varphi$ on both sides of the brane so $\varphi=-3 A$ everywhere.

Suppose now that there is a phase transition in the microphysics so that $V$ changes from one constant to another. Will the brane remain flat? We found that it is most easy to use the special bulk solution (IV.5) and (IV.6) to match onto the type II solution in KSS. By causality, the static solution must persist for the region of the bulk defined by $u=t-y<0$ and $v=t+y<0$. We thus take the solution (IV.1) for $B$ to be the following form

$$
B(u, v)=\frac{1}{3} \log \left(y_{*}(u)+u-v\right)
$$

for $y>0$ and, for $y<0$,

$$
B(u, v)=\frac{1}{3} \log \left(y_{*}(v)+v-u\right)
$$

In writing down ( $\nabla .11)$ and $(\overline{V .12})$ we have used the continuity condition of $B(u, v)$ at the location of the 3-brane $u=v \cdot y_{*}$ describes the time evolution of the location of the naked singularity on both sides. Before the phase transition, the universe is static and we have $y_{*}=$ constant. Given $B(u, v)$ we obtain $\varphi(u, v)$ and $A(u, v)$ for $y>0$ 


$$
\begin{aligned}
\varphi(u, v) & =\epsilon \log \left(y_{*}(u)+u-v\right)+d \\
A(u, v) & =\frac{1}{3} \log \left(y_{*}(u)+u-v\right)+\frac{1}{2} \log \left(1+y_{*}^{\prime}(u)\right)+a
\end{aligned}
$$

and for $y<0$

$$
\begin{aligned}
& \varphi(u, v)=\epsilon \log \left(y_{*}(v)+v-u\right)+d \\
& A(u, v)=\frac{1}{3} \log \left(y_{*}(v)+v-u\right)+\frac{1}{2} \log \left(1+y_{*}^{\prime}(v)\right)+a .
\end{aligned}
$$

$d$ and $a$ are integration constants and $\epsilon= \pm 1$. This solution was determined as follows. Demanding Poincare invariance on the brane for the initially static solution sets $A=B$ and thus $\alpha^{2}=9$ in ([V.6). The continuity condition for $\varphi(u, v)$ requires $\epsilon$ to take the same sign on both sides of the brane. (II.16) determines $b=-\epsilon \frac{4}{3}$. It is clear that, for $y_{*}=$ constant $>0$, this solution reduces to the type II static solution discussed in KSS.

Using the matching conditions (II.13) and ([1.14) we obtain

$$
\begin{aligned}
\rho_{T}(t) & =4 e^{-a-b d} \frac{y_{*}^{\prime}(t)+2}{\left|y_{*}^{\prime}(t)+1\right|^{\frac{1}{2}}}, \\
\rho_{T}(t)+P_{T}(t) & =-2 e^{-a-b d} \frac{y_{*}(t) y_{*}^{\prime \prime}(t)}{\left|y_{*}^{\prime}(t)+1\right|^{\frac{1}{2}}\left(y_{*}^{\prime}(t)+1\right)} .
\end{aligned}
$$

From $(\nabla .17)$ we see immediately that $\rho_{T}$ depends on $y_{*}^{\prime}$ only, but not on the value of $y_{*}$. This means that, in order for the vacuum energy density $V$ to settle to a constant value after the phase transition, $y_{*}^{\prime}(t)$ has to be a constant in the future.

There are two possible scenarios following from this observation. The first is that $y_{*}(t)$ settles to a constant value and thus $y_{*}^{\prime}=0$. (V.17) and (V.18) implies the vacuum energy density $V$ would always go back to its original value before the phase transition and the universe becomes static again. In this scenario the vacuum energy density is determined by the constants of integration $a$ and $d$ and is independent of the value of $y_{*}$.

The second scenario is that $y_{*}(t)$ becomes linear in $t$ for $t>0$. In this case, the vacuum energy density $V$ could change to another constant value. However, the universe is not static even after the phase transition and would start evolving thereafter. As an example we take $y_{*}(t)=y_{0}-\xi t$ with $0<\xi<1$, then the vacuum energy density changes to 


$$
\begin{aligned}
V_{2} & =4 e^{-a-b d} \frac{2-\xi}{\sqrt{1-\xi}} \\
& =\frac{V_{1}}{2} \frac{2-\xi}{\sqrt{1-\xi}},
\end{aligned}
$$

where $V_{1}$ is the initial value of the vacuum energy density. One can check that the transition can occur with positive energy density in the radiation. After the transition the universe is no longer static, but evolves toward a 'big crunch' singularity.

Here we have constructed an example which matches onto the initially static type II solution of KSS by using the special bulk solution (IV.5) and (IV.6). The continuity condition of $\varphi$ requires $\epsilon$ to take the same sign in $(\mathrm{V.13})$ and $(\mathrm{V.15})$. We are thus unable to match onto the type I solution of KSS using this special solution since the type I solution needs $\epsilon$ to take opposite sign. It would be interesting to try to use the general solutions in Section IV to match onto the type I solution of KSS and study scenarios with phase transitions.

\section{ACKNOWLEDGMENTS}

We thank Lisa Randall, Eva Silverstein and Erik Verlinde for informative discussions. We also would like to thank Daniel Holz for help making the figures. This work was supported in part by the NSF under grant numbers PHY89-04035 and PHY95-07065, and also by Department of Energy under grant number DOE-ER-40682-143. I. L. is supported in part by an ITP Graduate Fellowship. 


\section{REFERENCES}

[1] N. Arkani-Hamed, S. Dimopoulos and G. Dvali, Phys. Lett. B429 (1998) 263; I. Antoniadis, N. Arkani-Hamed, S. Dimopoulos and G. Dvali, Phys. Lett., B436 (1998) 257.

[2] L. Randall and R. Sundrum, Phys. Rev. Lett. 83 (1999) 3370; L. Randall and R. Sundrum, Phys. Rev. Lett. 83 (1999) 4690.

[3] J. de Boer, E. Verlinde and H. Verlinde, hep-th/9912012; E. Verlinde and H. Verlinde, hep-th/9912018; C. Schmidhuber, hep-th/9912156.

[4] S. Kachru, M. Schulz, and E. Silverstein, hep-th/0001206.

[5] N. Arkani-Hamed, S. Dimopoulos, N. Kaloper and R. Sundrum, hep-th/0001197.

[6] V. Rubakov and M. Shaposhnikov, Phys. Lett., B125 (1983) 139.

[7] I. Antoniadis, Phys. Lett. B246 (1990) 377.

[8] J. Overduin and P. Wesson, Phys. Rept. 283 (1997) 303; M Visser, hep-th/9910093, and references therein.

[9] S. Forste, Z. Lalak, S. Lavignac, and H.P. Nilles, hep-th/0002164; S.S. Gubser, hepth/0002160; S.P. de Alwis, hep-th/0002174; D. Youm, hep-th/0002147; J.W. Chen, M.A. Luty and E. Ponton, hep-th/0003067; I. Low and A. Zee, hep-th/0004124; S.P. de Alwis, A.T. Flournoy, and N. Irges, hep-th/0004125; C. Csaki, J. Erlich, C. Grojean, and T.J. Hollowood, hep-th/0004133.

[10] D. Amati and C. Klimcik, Phys. Lett. B219 (1989) 443.

[11] G.T. Horowitz and A.R. Steif, Phys. Rev. Lett. 64 (1990) 260; G.T. Horowitz and A.R. Steif, Phys. Rev. D42 (1990) 1950.

[12] E. Flanagan, H. Tye, I. Wasserman, hep-ph/9909373; C. Csaki, M. Graesser, L. Randall, J. Terning, hep-ph/9911406; S. Mukohyama, Phys. Lett. B473 (2000) 241; D. Ida, gr-qc/9912002; P. F. Gonzalez-Diaz, hep-th/0002033; R.N. Mohapatra, A. PerezLorenzana, C.A. de Sousa Pires, hep- ph/0003328; H. Stoica, H. Tye, I. Wasserman, hep-th/0004126; H. Kodama, A. Ishibashi, O. Seto, hep-th/0004160.

[13] S.W. Hawking and G.F.R. Ellis, The Large Scale Structure of Spacetime, Cambridge University Press (1973) p. 69.

[14] W. Israel, Nuovo Cim. 44B (1966) 1.

[15] R. Myers, Phys. Lett. B199 (1987) 371. 